\title{
D-dimer level for predicting the in-hospital mortality in liver cirrhosis: A retrospective study
}

\author{
YUN LI $^{1,2^{*}}$, XINGSHUN QI $^{1 *}$, HONGYU LI $^{1}$, JUNNA DAI $^{1,2}$, HAN DENG $^{1,2}$, JING LI $^{1,2}$, \\ YING PENG ${ }^{1,2}$, XU LIU ${ }^{1}$, XIAOLIN SUN ${ }^{1}$ and XIAOZHONG GUO ${ }^{1}$ \\ ${ }^{1}$ Liver Cirrhosis Study Group, Department of Gastroenterology, General Hospital of Shenyang Military Area, Shenyang, \\ Liaoning 110840; ${ }^{2}$ Postgraduate College, Dalian Medical University, Dalian, Liaoning 110016, P.R. China
}

Received August 29, 2015; Accepted September 22, 2016

DOI: $10.3892 /$ etm.2016.3930

\begin{abstract}
The present study aimed to examine the correlation of D-dimer levels with the Child-Pugh and MELD scores, as well as to determine the predictive ability of D-dimer level for the in-hospital mortality of liver cirrhosis patients. All cirrhotic patients who were consecutively admitted to our hospital between January 2011 and June 2014, and underwent D-dimer tests on admission were retrospectively analyzed. Pearson's $\chi^{2}$ tests were employed to evaluate the correlations of D-dimer levels with Child-Pugh and MELD scores. In addition, receiver operating curve (ROC) analysis was employed to evaluate the specificity and sensitivity of D-dimer levels for predicting the in-hospital mortality. In total, 703 cirrhotic patients were included in the study, with an in-hospital mortality of $5.4 \%$ (38/703). The D-dimer levels were correlated with Child-Pugh (correlation coefficient, 0.219; $\mathrm{P}<0.001$ ) and MELD scores (correlation coefficient, 0.207; $\mathrm{P}<0.001$ ). The highest D-dimer level was observed in the Child-Pugh class C patients, followed by the class B and A patients. Furthermore, D-dimer was significantly higher in the MELD score $>15$ group compared with the MELD score $<15$ group. The area under the ROC of D-dimer levels for predicting the in-hospital mortality of liver cirrhosis was $0.729(\mathrm{P}<0.0001)$, while the best cut-off D-dimer value was $0.28 \mu \mathrm{g} / \mathrm{ml}$ with a sensitivity of $86.84 \%$ and a specificity of $49.17 \%$. In conclusion, the D-dimer level is significantly associated with the degree of liver dysfunction. Therefore, D-dimer testing could be employed for the prognostic stratification of liver cirrhosis.
\end{abstract}

Correspondence to: Professor Hongyu Li or Dr Xingshun Qi, Liver Cirrhosis Study Group, Department of Gastroenterology, General Hospital of Shenyang Military Area, 83 Wenhua Road, Shenyang, Liaoning 110840, P.R. China

E-mail: 13309887041@163.com

E-mail: xingshunqi@126.com

*Contributed equally

Key words: D-dimer, Model for End-Stage Liver Disease, survival, Child-Pugh, liver cirrhosis

\section{Introduction}

D-dimer is a fibrin degradation product that is observed in the blood following clot degeneration. Currently, tests determining the concentration of D-dimer in the blood are widely employed in various clinical practices. Determination of D-dimer levels in combination with clinical probability assessment can be used to safely rule-out the diagnosis of pulmonary embolism (1-3). In addition, D-dimer levels are used for predicting the risk of recurrent venous thromboembolism and determining the duration of anticoagulation therapy in these patients (4-6).

The levels of D-dimer in the blood are significantly increased in patients with liver cirrhosis, and are gradually elevated further as the degree of liver dysfunction increases in severity $(7,8)$. It has been also suggested that D-dimer levels are influenced by the presence and treatment of ascites (9). Furthermore, the levels of this protein are significantly higher in cirrhotic patients with ascites compared with those without ascites, and are significantly decreased subsequently to ascite resolution (9). More recently, a meta-analysis performed by our group indicated that D-dimer levels are significantly associated with the presence of portal vein thrombosis in liver cirrhosis and may predict the development of portal vein thrombosis following splenectomy (10). Therefore, D-dimer may be a prognostic factor negatively associated with outcomes of liver cirrhosis.

In the present study, the correlation of D-dimer levels with the Child-Pugh and Model for End-Stage Liver Disease (MELD) scores was retrospectively analyzed, and determined the predictive ability of D-dimer levels for the in-hospital mortality in liver cirrhosis.

\section{Subjects and methods}

Study design. Patients consecutively admitted to the General Hospital of Shenyang Military Area (Shenyang, China) between January 2011 and June 2014 were retrospectively reviewed. The inclusion criteria were as follows: i) Patients were diagnosed with liver cirrhosis (based on clinical presentations, routine laboratory tests and imaging, and biopsy if necessary); and ii) D-dimer level was tested. The exclusion criteria were as follows: i) Patients were diagnosed with malignant tumors; ii) patients were diagnosed with venous or arterial thrombosis; iii) Child-Pugh and 
Table I. Patient characteristics $(n=703)$.

\begin{tabular}{|c|c|c|}
\hline Variables & Values & Reference range \\
\hline Mean age, years & $56.42 \pm 11.08$ & - \\
\hline \multicolumn{3}{|l|}{ Gender, $\mathrm{n}$} \\
\hline Male & $483(68.7 \%)$ & - \\
\hline Female & $220(31.3 \%)$ & - \\
\hline \multicolumn{3}{|l|}{ Etiology of liver cirrhosis, $n$} \\
\hline Viral hepatitis alone & $266(37.8 \%)$ & - \\
\hline Alcohol alone & $183(26.3 \%)$ & - \\
\hline Viral hepatitis + alcohol & $51(7.3 \%)$ & - \\
\hline Other or unknown & $203(28.9 \%)$ & - \\
\hline \multicolumn{3}{|l|}{ Ascites, $\mathrm{n}$} \\
\hline Absence & $381(54.2 \%)$ & - \\
\hline Presence & $322(45.8 \%)$ & - \\
\hline \multicolumn{3}{|l|}{ Hepatic encephalopathy, $\mathrm{n}$} \\
\hline Absence & $656(93.3 \%)$ & - \\
\hline Presence & $47(6.7 \%)$ & - \\
\hline Red blood cell count, $10^{12} / 1$ & $3.03 \pm 0.85$ & 4.3-5.8 \\
\hline Hemoglobin level, g/l & $91.76 \pm 29.19$ & $130-175$ \\
\hline White blood cell count, $10^{9} / 1$ & $5.71 \pm 4.84$ & $3.5-9.5$ \\
\hline Platelet level, $10^{9} / 1$ & $93.61 \pm 70.94$ & $135-350$ \\
\hline Total bilirubin, $\mu \mathrm{mol} / 1$ & $44.87 \pm 74.15$ & $5.1-22.2$ \\
\hline Albumin, g/l & $31.48 \pm 7.11$ & $40-55$ \\
\hline Alanine transaminase, $\mathrm{U} / 1$ & $42.69 \pm 14.85$ & $9-50$ \\
\hline Aspartate aminotransferase, U/1 & $76.94 \pm 468.48$ & $15-40$ \\
\hline Alkaline phosphatase, U/1 & $109.79 \pm 97.67$ & $45-125$ \\
\hline$\gamma$-glutamyl transferase, $\mathrm{U} / \mathrm{l}$ & $102.76 \pm 167.21$ & $10-60$ \\
\hline Creatinine, $\mu \mathrm{mol} / 1$ & $87.31 \pm 103.32$ & $44-133$ \\
\hline Prothrombin time, sec & $17.36 \pm 6.35$ & $11.5-14.5$ \\
\hline Activated partial prothrombin time, sec & $44.59 \pm 13.55$ & $28-40$ \\
\hline International normalized ratio & $1.53 \pm 1.91$ & - \\
\hline D-dimer, $\mu \mathrm{g} / \mathrm{ml}$ & $0.80 \pm 1.48$ & $0-0.3$ \\
\hline Child-Pugh score $^{\mathrm{a}}$ & $7.66 \pm 2.20$ & - \\
\hline MELD score & $8.63 \pm 8.14$ & - \\
\hline$>15$ & 570 & - \\
\hline$\leq 15$ & 133 & - \\
\hline \multicolumn{3}{|l|}{ In-hospital mortality, $\mathrm{n}$} \\
\hline No & $665(94.6 \%)$ & - \\
\hline Yes & $38(5.4 \%)$ & - \\
\hline
\end{tabular}

${ }^{a}$ The number of patients with Child-Pugh classes A, B and C were 249, 311 and 143, respectively. MELD, model for the end stage liver diseases.

MELD scores on admissions could not be calculated; and iv) the survival during their hospitalizations could not be evaluated. Repeated admissions were not excluded for patients meeting the aforementioned criteria. Certain of these patients have also been included in our previous studies (11-14). The present study was approved by the Ethics Committee of the General Hospital of Shenyang Military Area. Due to the retrospective nature of this study, the patients' written informed consents were waived.
Data collection. The following data were collected from the electronic medical records of the patients: Age, gender, etiology of liver diseases, presence of ascites, hepatic encephalopathy, red blood cell (RBC) count, hemoglobin ( $\mathrm{Hb}$ ) level, white blood cell (WBC) count, platelet level, total bilirubin (TBIL), albumin (ALB), alanine transaminase (ALT), aspartate aminotransferase (AST), alkaline phosphatate (ALP), $\gamma$-glutamine transferase (GGT), creatinine $(\mathrm{Cr})$, prothrombin time $(\mathrm{PT})$, 


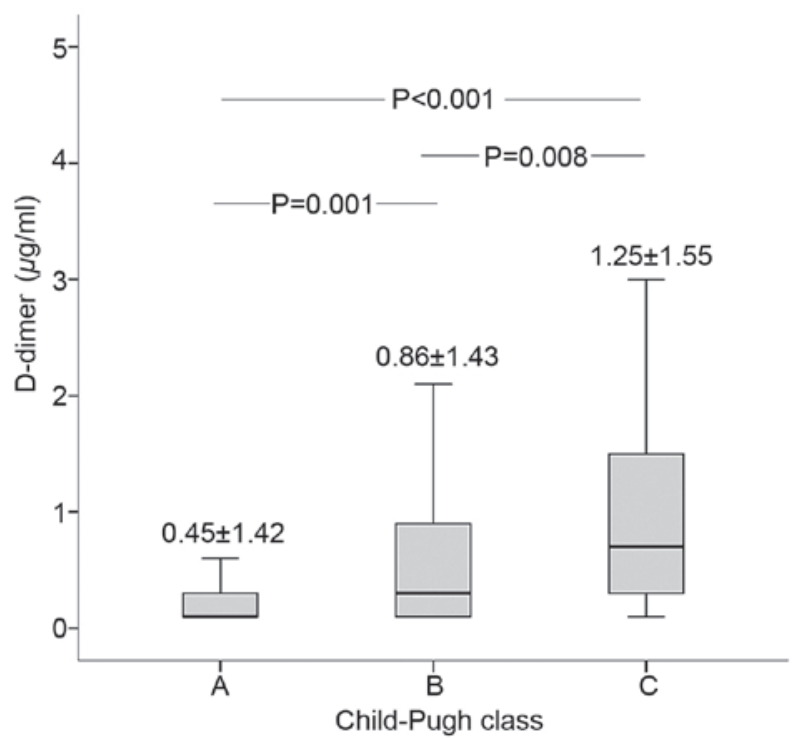

Figure 1. Boxplots demonstrating the comparison of D-dimer levels with the three Child-Pugh classifications (class A-C).

activated partial thromboplastin time (APTT), international normalized ratio (INR), D-dimer levels, Child-Pugh (15) and MELD scores (16), and in-hospital mortality. In cases where the D-dimer level was measured two or more times, the maximum $\mathrm{D}$-dimer value was selected for use in the current analysis.

Statistical analysis. Continuous data are presented as the mean \pm standard deviation, and were compared using the independent sample $t$ test or one-way analysis of variance. Categorical data are expressed as frequencies (i.e., rates of patients) and were compared using the $\chi^{2}$ test. The correlation of D-dimer levels with the Child-Pugh or MELD scores was evaluated by Pearson's $\chi^{2}$ test. Boxplots were also constructed to demonstrate the difference in the D-dimer levels among the various Child-Pugh classes (namely A-C) and MELD (namely scores $>15$ or $<15$ ). Receiver operating curve (ROC) analysis was employed to evaluate the specificity and sensitivity of D-dimer for predicting the in-hospital mortality. The optimal cut-off value was defined as the value at which the specificity plus sensitivity was maximal. In addition, the area under the ROC (AUROC) and the 95\% confidence intervals (CIs) were also calculated. A two-sided P-value of $<0.05$ was considered to indicate a statistically significant difference. All statistical analyses were performed using the MedCalc statistical software, version 15.10 (MedCalc Software bvba, Ostend, Belgium).

\section{Results}

Patients. During the enrollment period, a total of 703 patients were found to be eligible for inclusion in the current study. Patient characteristics are shown in Table I. The majority of patients enrolled into the present study were male (483/703 patients; $68.70 \%$ ). The most common etiology of liver cirrhosis was infection with hepatitis $\mathrm{B} / \mathrm{C}$ virus and/or alcohol consumption (500/703 patients; $71.12 \%)$. In addition,

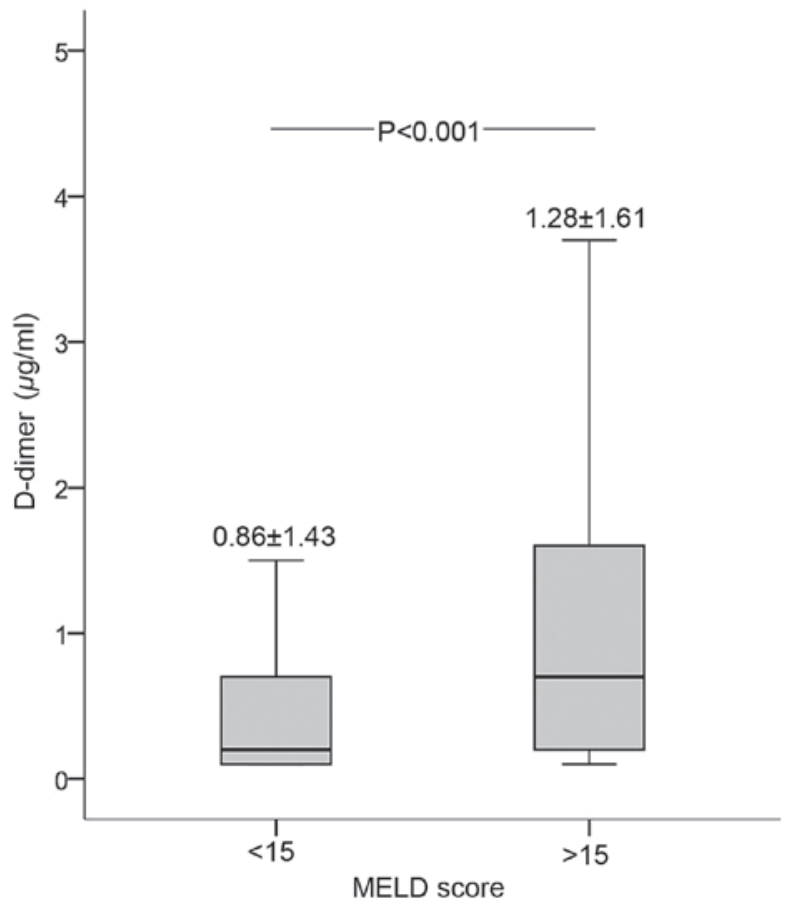

Figure 2. Boxplots demonstrating the comparison of D-dimer levels between the MELD score of $<15$ and $>15$ groups. MELD, Model for End-Stage Liver Disease.

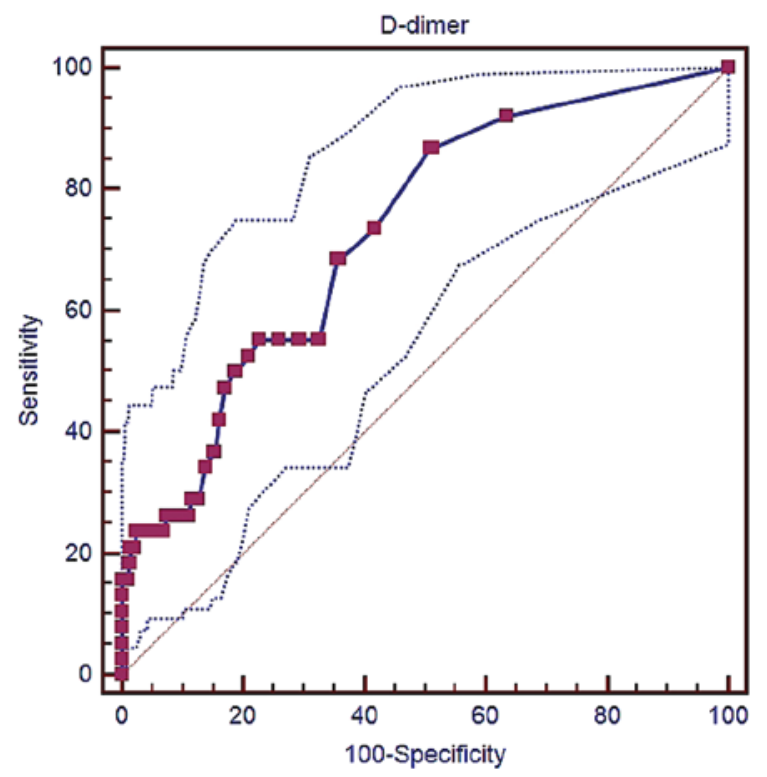

Figure 3. Receiver operating curve analysis of D-dimer levels for predicting the in-hospital mortality in liver cirrhosis patients.

the mean D-dimer level was $0.80 \pm 1.48 \mu \mathrm{g} / \mathrm{ml}$ (reference range, $0-0.3 \mu \mathrm{g} / \mathrm{ml})$. The mean MELD score was $8.63 \pm 8.14$. The mean Child-Pugh score was 7.66 \pm 2.20 . In-hospital mortality was found to be $5.4 \%$ (38/703 patients). The major causes of mortality included the following: Gastrointestinal bleeding $(n=13)$, progressive liver failure $(n=10)$, refractory hepatic encephalopathy $(n=6)$, multiple organ failure $(n=4)$, severe infection $(n=3)$, spontaneous bacterial peritonitis and hepatic encephalopathy $(\mathrm{n}=1)$ and pulmonary embolism $(\mathrm{n}=1)$. 
D-dimer and Child-Pugh score. The correlation of D-dimer levels with the Child-Pugh score of the patients was investigated by Pearson's $\chi^{2}$ test. The level of D-dimer was positively correlated with Child-Pugh score (correlation coefficient, 0.219 ; $\mathrm{P}<0.001)$. As shown in Fig. 1, D-dimer level was the highest in Child-Pugh class $\mathrm{C}$ group, followed by the Child-Pugh class B and then Child-Pugh class A groups. Furthermore, significant differences were observed between the different groups (C vs. $\mathrm{B}, \mathrm{P}=0.008$; $\mathrm{B}$ vs. $\mathrm{A}, \mathrm{P}=0.001$ ).

$D$-dimer and MELD score. The correlation of D-dimer levels with the Child-Pugh score of the patients was investigated by Pearson's $\chi^{2}$ test. As shown in Fig. 2, D-dimer level was positively correlated with the MELD score (correlation coefficient, 0.207; $\mathrm{P}<0.001)$. In addition, D-dimer level was significantly higher in the MELD score $>15$ group compared with that in the MELD score $<15$ group (Fig. 2).

D-dimer and in-hospital mortality. The specificity and sensitivity of D-dimer levels for predicting the in-hospital mortality of liver cirrhosis patients were determined, and the AUROC was calculated. As shown in Fig. 3, the AUROC of D-dimer level for this prediction was 0.729 (95\% CI, 0.695-0.762; $\mathrm{P}<0.0001)$. The best cut-off $\mathrm{D}$-dimer value was $0.28 \mu \mathrm{g} / \mathrm{ml}$, with a sensitivity of $86.84 \%$ and a specificity of $49.17 \%$.

\section{Discussion}

The present study identified that the D-dimer level of liver cirrhosis patients was significantly correlated with the Child-Pugh and MELD scores. This finding was largely consistent with several previous studies. For instance, an Egyptian study by El-Sayed et al investigated the D-dimer levels in 67 patients with chronic liver diseases and 30 healthy controls (17). The study observed that cirrhotic patients with Child-Pughclass A and B had significantly higherD-dimerlevels compared with the non-cirrhotic patients and healthy controls (class B, $147.32 \pm 114.16 \mathrm{ng} / \mathrm{ml}$; class A, $115.3 \pm 138.4 \mathrm{ng} / \mathrm{ml}$; non-cirrhotic liver disease, $28.86 \pm 40.03 \mathrm{ng} / \mathrm{ml}$; healthy controls, $17.6 \pm 11.7 \mathrm{ng} / \mathrm{ml}$ ). In addition, a Chinese study by Cong et al analyzed the D-dimer levels of 43 cirrhotic patients classified according to the Child-Pugh scores, as well as of 16 healthy controls (18). The D-dimer levels were demonstrated to gradually increase among Child-Pugh class A, B and C. In an Italian study, Violi et al also identified that the median D-dimer levels were $95.5,113$ and $1,453 \mathrm{ng} / \mathrm{ml}$ in patients with Child-Pugh class A, B and C, respectively (19). Furthermore, another Italian study by Primignani et al enrolled 43 cirrhotic patients with esophageal variceal bleeding and 43 cirrhotic patients without bleeding (20). In the patients with bleeding, the mean D-dimer levels were 127.38 $\pm 2.13,155.89 \pm 3.29$ and $432.3 \pm 2.9 \mathrm{ng} / \mathrm{ml}$ for Child-Pugh class A, B and C, respectively. By contrast, in the patients without bleeding, the mean D-dimer levels were $25.6 \pm 2.4,97.58 \pm 3.38$ and $246.36 \pm 2.65 \mathrm{ng} / \mathrm{ml}$ for Child-Pugh class A, B and C, respectively. Additionally, the mean D-dimer levels were significantly higher patients with bleeding that had a MELD score $>17$ compared with those having a MELD score $<17(486.5 \pm 3.22$ vs. $161.2 \pm 3.10$, respectively; $\mathrm{P}=0.01)(20)$. However, the authors did not observe any significant association of D-dimer levels with
MELD score in patients without bleeding (20). Collectively, the aforementioned studies supported the activation of fibrinolysis according to the severity of liver dysfunction. However, it must be acknowledged that the correlation between $\mathrm{D}$-dimer and the degree of liver dysfunction was relatively weak in the present study (correlation coefficient, $<0.3$ ).

Another finding of the current study was that higher D-dimer levels were able to significantly predict the in-hospital mortality in cirrhotic patients. Therefore, D-dimer testing may be used for the prognostic stratification of liver cirrhosis. Similarly, Primignani et al also compared the association of D-dimer levels with the 6-week mortality rate of cirrhotic patients with esophageal variceal bleeding (20). They identified that the mean D-dimer level was $172.9 \pm 2.70$ and $525.6 \pm 3.29 \mathrm{ng} / \mathrm{ml}$ in survivors and non-survivors, respectively. The proportion of hyperfibrinolysis, defined as a D-dimer level of $>483 \mathrm{ng} / \mathrm{ml}$, was 11 and $67 \%$ in survivors and non-survivors, respectively. In addition, the odds ratio of D-dimer level for predicting the 6-week mortality was 16 (20). These findings further supported the prognostic value of D-dimer levels in cirrhotic patients. By comparison, the current study further identified the accurate cut-off value in a more generalized population (with and without bleeding). However, considering that the AUROC was 0.729 in the present study, the prognostic value of D-dimer levels may be moderate.

A major limitation of the current study was its retrospective nature, which results in potential patient selection bias. However, considering that a relatively large number of patients were included in the study, the bias was weak.

In conclusion, the D-dimer levels of liver cirrhosis patients were found to be significantly associated with the degree of liver dysfunction. Furthermore, higher D-dimer levels predicted an increased risk of in-hospital mortality as a result of liver cirrhosis. Further prospective cohort studies are thus warranted to confirm the present findings.

\section{Acknowledgements}

This study was partially supported by a grant from the Natural Science Foundation of Liaoning Province (grant no. 2014020059) for Dr Hongyu Li.

\section{References}

1. Le Gal G, Righini M and Wells PS: D-dimer for pulmonary embolism. JAMA 313: 1668-1669, 2015.

2. Rodger MA, Le Gal G, Wells P, Baglin T, Aujesky D, Righini M, Palareti G, Huisman M and Meyer G: Clinical decision rules and D-Dimer in venous thromboembolism: Current controversies and future research priorities. Thromb Res 134: 763-768, 2014.

3. Geersing GJ, Janssen KJ, Oudega R, Bax L, Hoes AW, Reitsma JB and Moons KG: Excluding venous thromboembolism using point of care D-dimer tests in outpatients: A diagnostic meta-analysis. BMJ 339: b2990, 2009.

4. Palareti G, Legnani C, Cosmi B, Guazzaloca G, Pancani C and Coccheri S: Risk of venous thromboembolism recurrence: High negative predictive value of $\mathrm{D}$-dimer performed after oral anticoagulation is stopped. Thromb Haemost 87: 7-12, 2002.

5. Palareti G, Cosmi B, Legnani C, Antonucci E, De Micheli V, Ghirarduzzi A, Poli D, Testa S, Tosetto A, Pengo V, et al: D-dimer to guide the duration of anticoagulation in patients with venous thromboembolism: A management study. Blood 124: 196-203, 2014.

6. Palareti G, Cosmi B, Legnani C, Tosetto A, Brusi C, Iorio A, Pengo V, Ghirarduzzi A, Pattacini C, Testa S, et al: D-dimer testing to determine the duration of anticoagulation therapy. $\mathrm{N}$ Engl J Med 355: 1780-1789, 2006. 
7. Gram J,Duscha H,Zurborn KH and Bruhn HD: Increased levels of fibrinolysis reaction products (D-dimer) in patients with decompensated alcoholic liver cirrhosis. Scand J Gastroenterol 26 : 1173-1178, 1991.

8. Cioni G, Cristani A,Mussini C, Grandi S, Pentore R, Zeneroli ML, Tizzanini W, Zagni G and Ventura E: Incidence and clinical significance of elevated fibrin(ogen) degradation product and/or D-dimer levels in liver cirrhosis patients. Ital J Gastroenterol 22: 70-74, 1990.

9. Saray A, Mesihovic R, Gornjakovic S, Vanis N, Mehmedovic A, Nahodovic K, Glavas S and Papovic V: Association between high D-dimer plasma levels and ascites in patients with liver cirrhosis Med Arch 66: 372-374, 2012.

10. Dai J, Qi X, Li H and Guo X: Role of D-dimer in the development of portal vein thrombosis in liver cirrhosis: A meta-analysis. Saudi J Gastroenterol 21: 165-174, 2015.

11. Qi X, Li H, Chen J, Xia C, Peng Y, Dai J, Hou Y, Deng H, Li J and Guo X: Serum liver fibrosis markers for predicting the presence of gastroesophageal varices in liver cirrhosis: A retrospective cross-sectional study. Gastroenterol Res Pract 2015: 274534, 2015.

12. Peng Y,Qi X, Dai J,Li H and Guo X: Child-Pugh versus MELD score for predicting the in-hospital mortality of acute upper gastrointestinal bleeding in liver cirrhosis. Int J Clin Exp Med 8: 751-757, 2015.

13. Qi X, Peng Y, Li H, Li H and Guo X: Diabetes is associated with an increased risk of in-hospital mortality in liver cirrhosis with acute upper gastrointestinal bleeding. Eur J Gastroenterol Hepatol 27: 476-477, 2015.
14. Zhu C, Qi X, Li H, Peng Y, Dai J, Chen J, Xia C, Hou Y, Zhang $\mathrm{W}$ and Guo $\mathrm{X}$ : Correlation of serum liver fibrosis markers with severity of liver dysfunction in liver cirrhosis: A retrospective cross-sectional study. Int J Clin Exp Med 8: 5989-5998, 2015.

15. Pugh RN, Murray-Lyon IM, Dawson JL, Pietroni MC and Williams R: Transection of the oesophagus for bleeding oesophageal varices. Br J Surg 60: 646-649, 1973.

16. Kamath PS and Kim WR: The model for end-stage liver disease (MELD). Hepatology 45: 797-805, 2007.

17. El-Sayed R, El-Karaksy H, El-Raziky M, El-Hawary M, El Koofy N, Helmy H and Fahmy M: Assessment of coagulation and fibrinolysis in children with chronic liver disease. Blood Coagul Fibrinolysis 24: 113-117, 2013.

18. Cong YL, Wei YX, Zhang LW, Yin ZJ and Bai J: The relationship between hemostatic changes in liver cirrhosis patients with different degrees of liver lesions in reference to Child-Pugh scores. Zhonghua Gan Zang Bing Za Zhi 13: 31-34, 2005 (In Chinese).

19. Violi F, Ferro D, Basili S, Saliola M, Quintarelli C, Alessandri C and Cordova C: Association between low-grade disseminated intravascular coagulation and endotoxemia in patients with liver cirrhosis. Gastroenterology 109: 531-539, 1995.

20. Primignani M, Dell'Era A, Bucciarelli P, Bottasso B, Bajetta MT, de Franchis R and Cattaneo M: High-D-dimer plasma levels predict poor outcome in esophageal variceal bleeding. Dig Liver Dis 40: 874-881, 2008. 